\title{
Pitch-angle diffusion coefficients from resonant interactions with electrostatic electron cyclotron harmonic waves in planetary magnetospheres
}

\author{
A. K. Tripathi ${ }^{1}$, R. P. Singhal ${ }^{1}$, and K. P. Singh ${ }^{2}$ \\ ${ }^{1}$ Department of Applied Physics, Institute of Technology, Banaras Hindu University, Varanasi-221005, India \\ ${ }^{2}$ Department of Electronics Engg, Institute of Technology, Banaras Hindu University, Varanasi-221005, India
}

Received: 7 September 2010 - Revised: 8 December 2010 - Accepted: 8 December 2010 - Published: 11 February 2011

\begin{abstract}
Pitch-angle diffusion coefficients have been calculated for resonant interaction with electrostatic electron cyclotron harmonic $(\mathrm{ECH})$ waves in the magnetospheres of Earth, Jupiter, Saturn, Uranus and Neptune. Calculations have been performed at two radial distances of each planet. It is found that observed wave electric field amplitudes in the magnetospheres of Earth and Jupiter are sufficient to put electrons on strong diffusion in the energy range of less than $100 \mathrm{eV}$. However, for Saturn, Uranus and Neptune, the observed ECH wave amplitude are insufficient to put electrons on strong diffusion at any radial distance.
\end{abstract}

Keywords. Magnetospheric physics (Energetic particles, precipitating)

\section{Introduction}

Several spacecraft plasma wave instruments observed many familiar plasma waves during the encounter with the magnetospheres of Earth, Jupiter, Saturn, Uranus and Neptune (Gurnett et al., 1979a, b, 1996, 2005, 1989; Gurnett and Inan, 1988; Scarf et al., 1979; Kurth et al., 1980, 1983, 1987; Kurth and Gurnett, 1991; Zarka, 2004). Kurth and Gurnett, (1991) provided a comparative study of plasma waves of the planetary magnetospheres, for the first time. Recently, the comparative study of plasma waves at each outer planet has been reviewed by Zarka (2004). Electrostatic electron cyclotron harmonic $(\mathrm{ECH})$ waves are virtually ubiquitous in planetary magnetospheres. Resonant wave-particle interaction is the major source of both pitch-angle and energy diffusion of trapped electrons in the radiation belt (Kennel and Petschek, 1966; Summers et al., 2007, 2009). Summers et

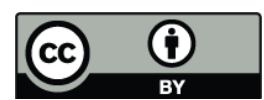

Correspondence to: A. K. Tripathi (aktrip2001@yahoo.co.in) al. (2007) present formulae for the bounce-averaged quasilinear diffusion coefficient for cyclotron resonance with field aligned electromagnetic waves in multi-ion plasma. Summers et al. (2009) reexamine the Kennel and Petschek (1966) concept of self limitation of stably trapped particle fluxes in a planetary magnetosphere. Pitch-angle diffusion and the resulting electron precipitation may produce the diffuse auroral emissions observed by different spacecraft in planetary magnetosphere (Kurth and Gurnett, 1991; Zarka, 2004). Several authors have modeled quasi-linear pitch-angle scattering due to ECH wave-particle interactions for these planets: Earth (Kennel et al., 1970; Lyons, 1974), Jupiter (Thorne, 1983), Saturn (Kurth et al., 1983), Uranus (Kurth et al., 1987) and Neptune (Gurnett et al., 1989). Strong pitch-angle diffusion is the process for scattering plasma sheet electrons into the polar atmosphere of the planets to produce the diffuse aurora. Electrostatic waves rather than electromagnetic waves are believed to be the scattering mechanism of the plasma sheet electrons because in the presence of cold plasma, electrostatic waves propagate at arbitrarily small phase velocities, making it possible to interact resonantly with much slower electrons. However, the dominant scattering process for diffuse auroral precipitation has not yet been definitively identified though several physical mechanisms have been suggested.

Horne and Thorne (2000) have calculated the bounceaveraged pitch-angle diffusion rates for electrostatic ECH waves driven by a loss-cone distribution. They have concluded that ECH waves can scatter $\mathrm{keV}$ electrons into the loss-cone efficiently and hence can contribute directly to the loss to the atmosphere. Detailed quasi-linear scattering rates, based on observed wave spectral properties, demonstrated that ECH waves are capable of causing strong diffusion scattering of electrons $(\sim 1 \mathrm{keV})$ near the loss-cone (Horne et al., 2003). Recently, Ni et al. (2008) analysed the resonating scattering of plasma sheet electrons $(\sim 100 \mathrm{eV}-20 \mathrm{keV})$ at $L=6$ due to resonant interactions with whistler-mode chorus 
waves. The authors have found that scattering by chorus waves is more dominant than scattering by ECH waves. Thus, chorus scattering is a major contributor to the origin of the diffuse aurora. In this paper, we extend the calculations of pitch-angle diffusion coefficients for resonant interaction with ECH waves in the magnetospheres of Earth, Jupiter, Saturn, Uranus and Neptune. This will provide a comparative study of pitch-angle diffusion in producing diffuse aurora in planetary atmospheres for the first time.

The paper is organized in the following manner: Sect. 2 describes the detailed observations of $\mathrm{ECH}$ emissions in planetary magnetospheres. In Sect. 3, we present the dispersion relation for $\mathrm{ECH}$ waves propagating perpendicular to the ambient magnetic field considering linear wave theory analysis. Expressions for pitch-angle diffusion and strong diffusion coefficients are also presented in this section. In Sect. 4, observations of plasma parameters appropriate for particular radial distance in magnetospheres and used in present study are discussed. This section also describes the numerical procedure for calculating the temporal growth rates of ECH waves. The comparison of pitch-angle diffusion with strong diffusion and precipitation of energetic electrons into the atmosphere of each planet and results and discussion of this study, are presented in Sect. 5. Finally, in Sect. 6, we present the conclusions.

\section{Observations of ECH waves}

\subsection{Earth}

The ECH waves of natural origin were first observed by the OGO-5 satellite (Kennel et al., 1970; Fredricks and Scarf, 1973) as narrow band $\left(\Delta f / f_{\mathrm{c}} \approx 1.0\right)$, large amplitude $\left(1-10 \mathrm{mV} \mathrm{m}^{-1}\right)$ waves that appear within a frequency range of $1.25 f_{\mathrm{c}}<f<1.75 f_{\mathrm{c}}$. Similar $3 / 2$ emissions have also been observed by IMP-6 (Shaw and Gurnett, 1975) satellites. At larger radial distances, from about 17:30 to 19:15 UT, the $(n+1 / 2) f_{\mathrm{c}}$ electrostatic emissions at $(3 / 2) f_{\mathrm{c}}$ and $(5 / 2) f_{\mathrm{c}}$ become much more intense ranging from several hundred $\mu \mathrm{V} \mathrm{m}^{-1}$ to about one $\mathrm{mV} \mathrm{m}^{-1}$ (Gurnett et al., 1979a). In recent years, Meredith et al. (2000) reported ECH intensities $10^{-(6.7 \pm 1.2)} \mathrm{V}^{2} \mathrm{~m}^{-2}$ outside $L=6.0$ and $10^{-(6.9 \pm 0.9)} \mathrm{V}^{2} \mathrm{~m}^{-2}$ inside $L=6.0$ observed by instruments onboard the CRRES spacecraft. Décréau et al. (2001) described the observations of ECH wave intensities observed by WHISPER instrument onboard the CLUSTER spacecraft. Intense $3 / 2 f_{\text {ce }}\left(\geq 1.5 \mathrm{mV} \mathrm{m}^{-1}\right)$ emissions are observed with higher proportion during CLUSTER spacecraft perigee $\left(4 R_{\mathrm{E}}\right)$ passes in the nightside sector compared to geostationary spacecraft (GEOS) studies. Intensity of equatorial ECH emissions increases with geocentric distance. The emissions are observed most often in the radial distance from 4 to $8 R_{\mathrm{E}}$ and magnetic latitude in the range $\pm 10^{\circ}$.

\subsection{Jupiter}

The Voyagers 1 and 2 encounters with Jupiter have provided us with the first comprehensive investigation of plasma waves in the Jovian magnetosphere. The Voyager 1 plasma wave instrument detected for the first time electrostatic emissions related to the electron gyrofrequency beyond the boundary of the torus and near the magnetic equator crossings (Scarf et al., 1979). The secondary plasma wave observations were provided by the Voyager 2 spacecraft (Gurnett et al., 1979b). The intense electrostatic emissions near half-integral harmonics of the electron gyrofrquency, $3 f_{\mathrm{c}} / 2$, $5 f_{\mathrm{c}} / 2$, and intense narrowband emissions at $f_{\mathrm{UHR}}$ were observed when Voyager 2 crossed magnetic equator regions between $6<L<15$ at the geomagnetic latitude of $13^{\circ}$. The intensity of ECH bands at Jupiter is typically $100 \mu \mathrm{V} \mathrm{m}^{-1}$ with occasional bursts to a few millivolts per meter. The intensities of the emissions are greatest at smaller radial distances and decrease with increasing distance from the centre of planet (Kurth et al., 1980; Kurth and Gurnett, 1991). During the Ulysses and Galileo encounters with Jupiter, intense emissions were recorded near $(3 / 2) f_{\text {ce }}$, and $(5 / 2) f_{\text {ce }}$ when the spacecraft crossed the magnetic equator in the Io torus (Stone et al., 1992; Gurnett et al., 1996).

\subsection{Saturn}

The first evidence for electrostatic waves in the Saturnian magnetosphere was given by Gurnett et al., (1981). They reported a series of ECH banded emissions or $(n+1 / 2) f_{\text {ce }}$ emissions on the Voyager 1 inbound leg. The $3 / 2 f_{\text {ce }}$ bands were observed on the Voyager 1 outbound leg inside $\sim 7 R_{\mathrm{S}}$ (where $R_{\mathrm{S}}$ is radius of the Saturn). The Voyager 2 encounter offered a second look at electrostatic waves in Saturn's magnetosphere. Scarf et al. (1982) again reported $(n+1 / 2) f_{\text {ce }}$ emissions throughout the inner magnetosphere lying in the range of $4<R<8 R_{\mathrm{S}}$. The intensities are typically $30 \mu \mathrm{V} \mathrm{m}^{-1}$, less intense than those at Earth and three times smaller than Jupiter. Recently, Gurnett et al. (2005) presented the first results of observed plasma waves from Cassini radio and plasma wave science (RPWS) instrument during the approach and first orbit around Saturn. They reported that the strongest ECH emissions were observed in the inner region of the magnetosphere, inside of $\sim 10 R_{S}$.

\subsection{Uranus}

ECH emissions from Uranus were detected by the the Voyager 2 plasma wave instruments in the radial distance range $\sim 12 R_{\mathrm{U}}$ (where $R_{\mathrm{U}}$ is radius of Uranus). The intensities are comparable to those for Earth and Saturn, but somewhat weaker than the Jovian ECH waves. The ECH waves have intensities of only about $10 \mu \mathrm{V} \mathrm{m}^{-1}$. With such low intensities, strong pitch-angle scattering is not likely. However, experience with other planets suggests the intensities of the 
ECH emissions increase at smaller radial distances, presumably because the flux of resonant electrons would be greater closer to the planet (Kurth et al., 1987; Kurth and Gurnett, 1991).

\subsection{Neptune}

The first unambiguously-identified plasma waves observed by Voyager 2 for Neptune were ECH emissions (Gurnett et al., 1989; Kurth and Gurnett, 1991). The ECH bands are not very intense and only reach about $30 \mu \mathrm{V} \mathrm{m}^{-1}$ (Kurth and Gurnett, 1991). The low intensity of the observed ECH emissions implies that they are unlikely candidates for strong pitch-angle scattering. It is possible that waves of significantly greater intensities occur at intermediate radial distances $10>R>2 R_{\mathrm{N}}$.

\section{Dispersion relation, pitch-angle diffusion and strong diffusion}

The expression of dispersion relation for electrostatic waves propagating perpendicular to the ambient magnetic field using linear theory analysis in hot magnetized plasma was given by Summers and Thorne (1995). We consider the distribution function as a combination of cold (Maxwellian) and hot components represented by loss-cone kappa distribution since the ECH waves are deriven unstable by a loss-cone feature. We write a Maxwellian for thermal component, $f_{\mathrm{M}}$, and kappa distribution with loss-cone, $f_{\kappa}$, for hot component introduced by Summers and Thorne (1995). Using quasilinear theory, Lyons (1974) first calculated bounce-averaged diffusion coefficients of electrostatic $1.5 \Omega_{\text {ce }}$ waves for scattering in both pitch-angle and energy. Recently, the bounceaveraged pitch-angle diffusion coefficients of electrons in detail have been calculated by Tripathi and Singhal (2009). The expression for the bounce- averaged diffusion rate, is given as

$$
\left\langle D_{\alpha \alpha}\right\rangle=T_{\text {frac }} D_{\alpha \alpha}
$$

with

$$
T_{\mathrm{frac}}=4 L R p \lambda / v \cos \alpha_{\mathrm{eq}} T_{\mathrm{b}}
$$

and

$T_{\mathrm{b}}=0.117\left(R_{o} / R_{\mathrm{p}}\right) c / v\left[1-0.4635\left(\sin \alpha_{\mathrm{eq}}\right)^{3 / 4}\right]$

where $T_{\text {frac }}$ is the fraction of time the particle interacts with the wave during one bounce period and $D_{\alpha \alpha}$ is evaluated at the magnetic equator. $\alpha_{\mathrm{eq}}$ is the equatorial pitch-angle and $T_{\mathrm{b}}$ is the particle bounce time for one complete cycle between the particle mirror points. $R_{O}$ is the distance from the centre of planet to the equatorial crossings points of a magnetic field line, $R_{\mathrm{p}}$ is the planet radius, $v$ is the particle velocity, and $c$ is the speed of light. The limit of strong diffusion is reached when the diffusion coefficient at the edge of the loss-cone is large to scatter particles across the loss-cone in less than a quarter of the bounce period $\left\langle D_{\alpha \alpha}\left(\alpha_{\mathrm{LC}}\right)\right\rangle \geq 4 \alpha_{\mathrm{LC}}^{2} / T_{\mathrm{b}}$ (Lyons, 1974; Horne and Thorne, 2000).

\section{Plasma parameters}

The present section describes the observations of basic plasma parameters measured in the magnetospheres of Earth, Jupiter, Saturn, Uranus, and Neptune and used in the present studies.

\subsection{Earth}

The observational data for electrons have been obtained by several spacecraft during encounters with Earth's magnetosphere (Olsen et al., 1987; Laakso et al., 2002). Recently, Laakso et al. (2002) reported the electron density values 100$0.1 \mathrm{~cm}^{-3}$ measured by polar satellite at $L=3 \sim 12$ and somewhat lower values at $L>12$. At the plasma pause, the density rapidly declines by a few orders of magnitude over a relatively short distance. During the dayside, the density is usually a few electrons per $\mathrm{cm}^{-3}$. The ratio of $n_{\mathrm{h}} / n_{\mathrm{c}}$ increases as the radial distance increases.

\subsection{Jupiter}

The Voyager's encounter with Jupiter provided detailed knowledge of the plasma properties of the magnetosphere (Belcher, 1983; Bagenal, 1994). The cold electron density $\left(n_{\mathrm{c}}\right)$ changes from about 2500 to $3.0 \mathrm{~cm}^{-3}$ as radial distance increases from $6 R_{\mathrm{J}}$ to $17 R_{\mathrm{J}}$. In the same range of radial distance, cold electron temperature $\left(T_{\mathrm{C}}\right)$ changes from 5 to $20 \mathrm{eV}$, hot temperature $\left(T_{\mathrm{h}}\right)$ from 150 to $1000 \mathrm{eV}$ and ratio of $n_{\mathrm{h}} / n_{\mathrm{c}}$ from $0.0001 \%$ to $0.3 \%$. Galileo spacecraft received the observational data during its passing through the torus (Bagenal et al., 1997). At $6 R_{\mathrm{J}}$, the measured electron density was maximum, i.e., $3775 \mathrm{~cm}^{-3}$ (Bagenal et al., 1997).

\subsection{Saturn}

The first in situ measurements of the plasma parameters in Saturn's inner magnetosphere were made by Voyager 1 and 2 during flybys of the planet (Sittler et al., 1983; Richardson and Sittler, 1990). The most recent plasma measurements in Saturn's magnetosphere have been made by the Cassini spacecraft, which was placed in orbit around Saturn (Gurnett et al., 2005). The value of electron $n_{\mathrm{c}}$ varies in the range of 200 to $0.50 \mathrm{~cm}^{-3}$ as the radial distance increases from $3.5 R_{\mathrm{S}}$ to $10 R_{\mathrm{S}}$. In the same range of distance, $T_{\mathrm{c}}$ changes in between $2 \mathrm{eV}$ to $10 \mathrm{eV}, T_{\mathrm{h}}$ ranges from $40 \mathrm{eV}$ to $360 \mathrm{eV}$ and $n_{\mathrm{h}} / n_{\mathrm{c}}$ from $0.03 \%$ to $0.5 \%$ (Gurnett et al., 2005).

\subsection{Uranus}

Sittler et al. (1987) have presented results of an analysis of the Voyager 2 PLS electron measurements made during the 
Table 1. Plasma parameters at different distances.

\begin{tabular}{lcccccc}
\hline Planet & Distance & $\begin{array}{c}n_{\mathrm{c}} \\
\left(\mathrm{cm}^{-3}\right)\end{array}$ & $\begin{array}{c}n_{\mathrm{h}} \\
\left(\mathrm{cm}^{-3}\right)\end{array}$ & $\begin{array}{c}T_{\mathrm{c}} \\
(\mathrm{eV})\end{array}$ & $\begin{array}{c}T_{\mathrm{h}} \\
(\mathrm{eV})\end{array}$ & $\begin{array}{c}B_{o} \\
(\mathrm{nT})\end{array}$ \\
\hline Earth & $R=4 R_{\mathrm{E}}$ & 35.0 & 1.75 & 10 & 400 & 386 \\
& $R=8 R_{\mathrm{E}}$ & 1.0 & 0.1 & 15 & 1000 & 58 \\
Jupiter & $R=6 R_{\mathrm{J}}$ & 3775 & 188.75 & 8 & 175 & 2007 \\
& $R=17 R_{\mathrm{J}}$ & 3.0 & 0.3 & 20 & 1000 & 51 \\
Saturn & $R=5 R_{\mathrm{S}}$ & 24.0 & 1.2 & 5 & 200 & 160 \\
& $R=8 R_{\mathrm{S}}$ & 0.75 & 0.075 & 8 & 300 & 36 \\
Uranus & $R=5 R_{\mathrm{U}}$ & 0.95 & 0.048 & 25 & 300 & 88 \\
& $R=11 R_{\mathrm{U}}$ & 0.05 & 0.005 & 65 & 1500 & 11 \\
Neptune & $R=5 R_{\mathrm{N}}$ & 0.60 & 0.03 & 10 & 160 & 72 \\
& $R=10 R_{\mathrm{N}}$ & 0.03 & 0.003 & 20 & 300 & 14 \\
\hline
\end{tabular}

Uranus encounter. Within the inner magnetosphere $(L>5)$ the electron density ranged between 0.02 and $1.0 \mathrm{~cm}^{-3}$, and temperatures ranged from $T_{\mathrm{e}} \sim 10 \mathrm{eV}$ near the inbound pass to $T_{\mathrm{e}} \sim 100 \mathrm{eV}$ within the nightside hemisphere (outbound pass). The electron $T_{\mathrm{c}}$ was $\sim 8-25 \mathrm{eV}$ near inbound pass and $T_{\mathrm{c}} \sim 10-70 \mathrm{eV}$ within the outbound pass. The ratio $n_{\mathrm{h}} / n_{\mathrm{c}}$ of the electrons ranged between $0.03 \%$ to $0.5 \%$ with temperature $T_{\mathrm{h}}$ ranging from $20 \mathrm{eV}$ to $200 \mathrm{eV}$ near the terminator (inbound pass) and $500 \mathrm{eV}$ to $2 \mathrm{keV}$ in the nightside region (outbound pass).

\subsection{Neptune}

The plasma science experiment on Voyager 2 made observations of the plasma parameters in Neptune's magnetosphere (Belcher et al., 1989; Zhang et al., 1991). The maximum electron density and temperature are $2 \mathrm{~cm}^{-3}$ and $300 \mathrm{eV}$, respectively. When cold electrons are present, hot electrons are a small fraction of the total population; the ratio $n_{\mathrm{h}} / n_{\mathrm{c}}$ is usually a few percent (Zhang et al., 1991). For radial distances from 5 to $10 R_{\mathrm{N}}$, the value of $n_{\mathrm{c}}$ varies between $\sim 0.60$ to $0.03 \mathrm{~cm}^{-3}, T_{\mathrm{c}}$ ranges from 9 to $20 \mathrm{eV}$ and $T_{\mathrm{h}}$ from $150 \mathrm{eV}$ to $400 \mathrm{eV}$.

\section{Calculation details}

Temporal growth rates have been calculated at two radial distances with spectral index, $\kappa=2$ and loss-cone index, $\ell=1$ for each planet using the plasma parameters given in Table 1. A numerical procedure for solving the ECH dispersion relation is used. Electron pitch-angle diffusion coefficients have been calculated using the representative growth rate profiles at particular radial distance of each planet (Tripathi and Singhal, 2009). Subscript "c" and " $h$ " are used for representing cold and hot electrons. The present calculations have considered the harmonics of first band, i.e., $1.5 \Omega_{\text {ce }}$ at both distances of each planet. We assume wave growth is centered at a propagation angle of $\psi=89^{\circ}$ and at Earth, wave electric field $\boldsymbol{E}_{\mathrm{W}}=1 \mathrm{mV} \mathrm{m}^{-1}$ for $L=4$, and $\boldsymbol{E}_{\mathrm{W}}=0.1 \mathrm{mV} \mathrm{m}^{-1}$ for

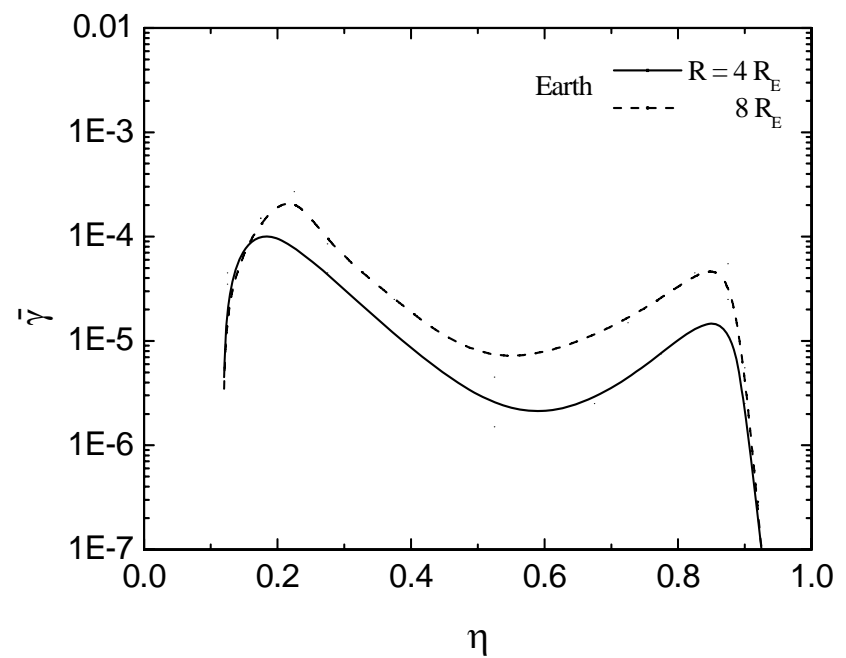

Fig. 1. Normalized temporal growth rates $\bar{\gamma}\left(=\gamma / \Omega_{\text {ce }}\right)$ versus frequency of ECH waves. The frequency within each band is $\bar{\omega}\left(=\omega_{r} / \Omega_{\mathrm{ce}}\right)=n+\eta$, where $n=1,2,3$ and $\eta$ lies between 0 and 1. Other plasma parameters are given in Table 1 .

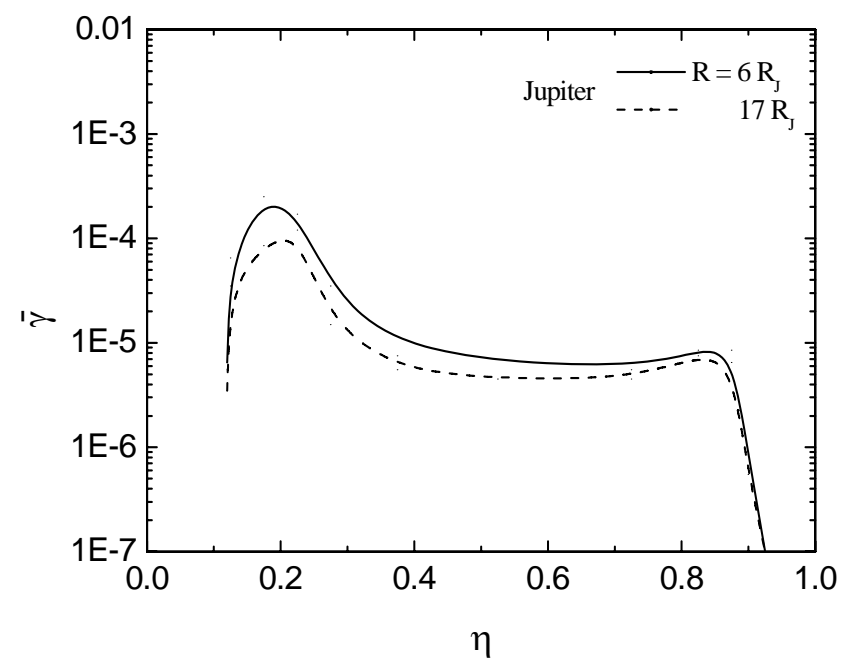

Fig. 2. Same as in Fig. 1 but for Jupiter.

$L=8$ is taken. In Jupiter $\boldsymbol{E}_{\mathrm{W}}=1 \mathrm{mV} \mathrm{m}^{-1}$ for $L=6$, and $\boldsymbol{E}_{\mathrm{W}}=0.1 \mathrm{mV} \mathrm{m}^{-1}$ for $L=17$ is considered. In case of Saturn, Uranus and Neptune we chose the value of $\boldsymbol{E}_{\mathrm{W}}=0.030$ at lower L-shell and $\boldsymbol{E}_{\mathrm{W}}=0.010 \mathrm{mV} \mathrm{m}^{-1}$ at higher L-shell.

\section{Results and discussion}

In Figs. 1-6 we show the temporal growth rate as a function of frequency for the first harmonic band at two radial distances for Earth, Jupiter, Saturn, Uranus and Neptune, respectively. These profiles of growth rates have been used to represent the wave intensity as a function of wave frequency. Figures 6-15 represent the variation of bounce-averaged 
Table 2. Diffusion rates for several electron energies at different distances.

\begin{tabular}{|c|c|c|c|c|c|c|c|c|}
\hline Planet & Distance & $\omega_{\mathrm{p}} / \Omega_{\mathrm{e}}$ & $\begin{array}{l}\alpha_{\mathrm{LC}} \\
\text { (degree) }\end{array}$ & $\begin{array}{l}\mathrm{E} \\
(\mathrm{eV})\end{array}$ & $\begin{array}{l}<D_{\alpha \alpha}> \\
\left(\mathrm{s}^{-1}\right)\end{array}$ & $\begin{array}{l}D_{\mathrm{SD}} \\
\left(\mathrm{s}^{-1}\right)\end{array}$ & $\begin{array}{l}E_{\mathrm{W} \mathrm{obs}} \\
\mathrm{mV} \mathrm{m}^{-1}\end{array}$ & $\begin{array}{l}E_{\mathrm{W} \text { req }} \\
\left(\mathrm{mV} \mathrm{m}^{-1}\right)\end{array}$ \\
\hline Earth & $L=4(8)$ & $4.9(5.7)$ & $5(2)$ & $\begin{array}{l}25 \\
50 \\
100 \\
200\end{array}$ & $\begin{array}{l}5.0 \times 10^{-3}\left(3.0 \times 10^{-5}\right) \\
7.0 \times 10^{-4}\left(7.5 \times 10^{-5}\right) \\
2.5 \times 10^{-4}\left(7.0 \times 10^{-6}\right) \\
8.6 \times 10^{-5}\left(1.1 \times 10^{-6}\right)\end{array}$ & $\begin{array}{l}7.1 \times 10^{-4}\left(4.3 \times 10^{-5}\right) \\
1.0 \times 10^{-3}\left(6.1 \times 10^{-5}\right) \\
1.4 \times 10^{-3}\left(8.5 \times 10^{-5}\right) \\
2.1 \times 10^{-3}\left(1.2 \times 10^{-4}\right)\end{array}$ & $1.0(0.1)$ & $\begin{array}{l}0.40(0.12) \\
1.20(0.09) \\
2.40(0.35) \\
4.90(1.0)\end{array}$ \\
\hline Jupiter & $L=6(17)$ & $9.9(11)$ & $3(1)$ & $\begin{array}{l}25 \\
50 \\
100 \\
200\end{array}$ & $\begin{array}{l}10 \times 10^{-4}\left(2.7 \times 10^{-5}\right) \\
2.1 \times 10^{-4}\left(3.7 \times 10^{-6}\right) \\
5.2 \times 10^{-5}\left(9.7 \times 10^{-6}\right) \\
----\end{array}$ & $\begin{array}{l}1.4 \times 10^{-4}\left(2.0 \times 10^{-6}\right) \\
1.9 \times 10^{-4}\left(2.9 \times 10^{-6}\right) \\
2.5 \times 10^{-4}\left(4.2 \times 10^{-6}\right) \\
----\end{array}$ & $1.0(0.1)$ & $\begin{array}{l}0.40(0.03) \\
1.0(0.09) \\
2.20(0.07) \\
----\end{array}$ \\
\hline Saturn & $L=5(8)$ & $9.8(7.6)$ & $4(2)$ & $\begin{array}{l}25 \\
50 \\
100 \\
200\end{array}$ & $\begin{array}{l}4.4 \times 10^{-5}\left(6.0 \times 10^{-6}\right) \\
5.0 \times 10^{-6}\left(2.6 \times 10^{-6}\right) \\
2.9 \times 10^{-6}\left(1.4 \times 10^{-6}\right) \\
----\end{array}$ & $\begin{array}{l}2.9 \times 10^{-4}\left(4.3 \times 10^{-5}\right) \\
4.1 \times 10^{-4}\left(6.1 \times 10^{-5}\right) \\
5.9 \times 10^{-4}\left(8.7 \times 10^{-5}\right) \\
----\end{array}$ & $0.03(0.01)$ & $\begin{array}{l}0.08(0.027) \\
0.27(0.048) \\
0.42(0.079) \\
----\end{array}$ \\
\hline Uranus & $L=5(11)$ & $3.7(6.4)$ & $4(1)$ & $\begin{array}{l}25 \\
50 \\
100 \\
200\end{array}$ & $\begin{array}{l}6.4 \times 10^{-7}\left(4.2 \times 10^{-7}\right) \\
4.5 \times 10^{-7}\left(2.3 \times 10^{-7}\right) \\
1.9 \times 10^{-7}\left(1.5 \times 10^{-7}\right) \\
----\end{array}$ & $\begin{array}{l}3.0 \times 10^{-4}\left(1.5 \times 10^{-5}\right) \\
4.1 \times 10^{-4}\left(1.7 \times 10^{-5}\right) \\
5.9 \times 10^{-4}\left(2.4 \times 10^{-5}\right) \\
----\end{array}$ & $0.03(0.01)$ & $\begin{array}{l}0.65(0.06) \\
0.9(0.086) \\
1.70(0.13) \\
----\end{array}$ \\
\hline Neptune & $L=5(10)$ & $3.4(4.0)$ & $4(1)$ & $\begin{array}{l}25 \\
50 \\
100 \\
200\end{array}$ & $\begin{array}{l}6.1 \times 10^{-7}\left(4.2 \times 10^{-6}\right) \\
3.2 \times 10^{-7}\left(6.1 \times 10^{-7}\right) \\
3.0 \times 10^{-7}\left(9.5 \times 10^{-8}\right) \\
----\end{array}$ & $\begin{array}{l}2.8 \times 10^{-4}\left(1.7 \times 10^{-5}\right) \\
4.0 \times 10^{-4}\left(2.5 \times 10^{-5}\right) \\
5.7 \times 10^{-4}\left(6.9 \times 10^{-5}\right) \\
----\end{array}$ & $0.03(0.01)$ & $\begin{array}{l}0.64(0.02) \\
1.10(0.064) \\
1.30(0.27) \\
----\end{array}$ \\
\hline
\end{tabular}

$\alpha_{\mathrm{LC}}$ is loss-cone angle at particular height

$E_{\mathrm{W} \text { obs }}$ is magnitude of observed electric field at particular radial distance

$E_{\mathrm{W} \text { req }}$ is magnitude of required electric field for strong diffusion at particular radial distance

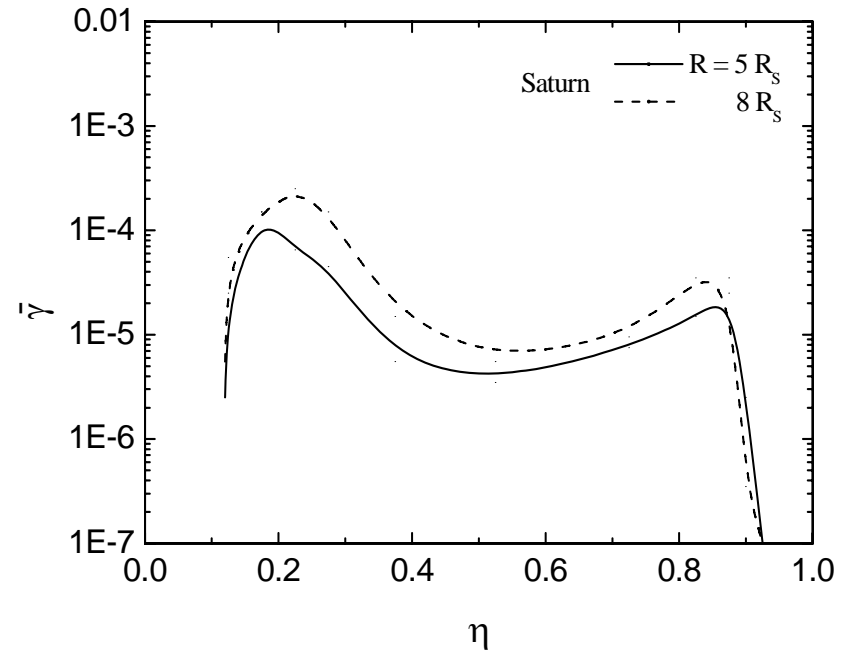

Fig. 3. Same as in Fig. 1 but for Saturn.

pitch angle diffusion rates as a function of equatorial pitch angle for the above planets at two radial distances for each planet. In the case of Earth and Jupiter it is noted that the dif-

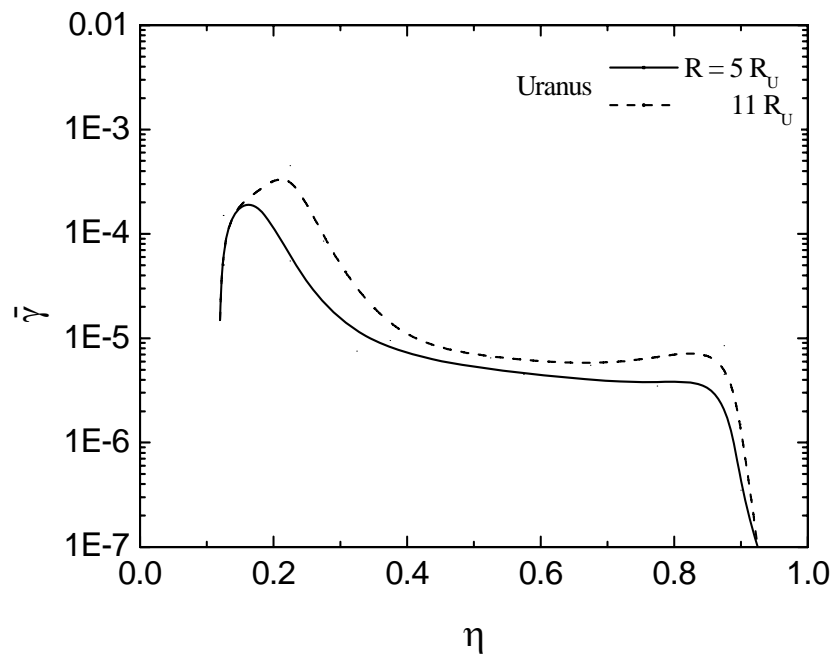

Fig. 4. Same as in Fig. 1 but for Uranus.

fusion rate decreases by several orders of magnitude as the radial distance increases. Further, the diffusion rates drop more rapidly with an increasing pitch angle for all energies 


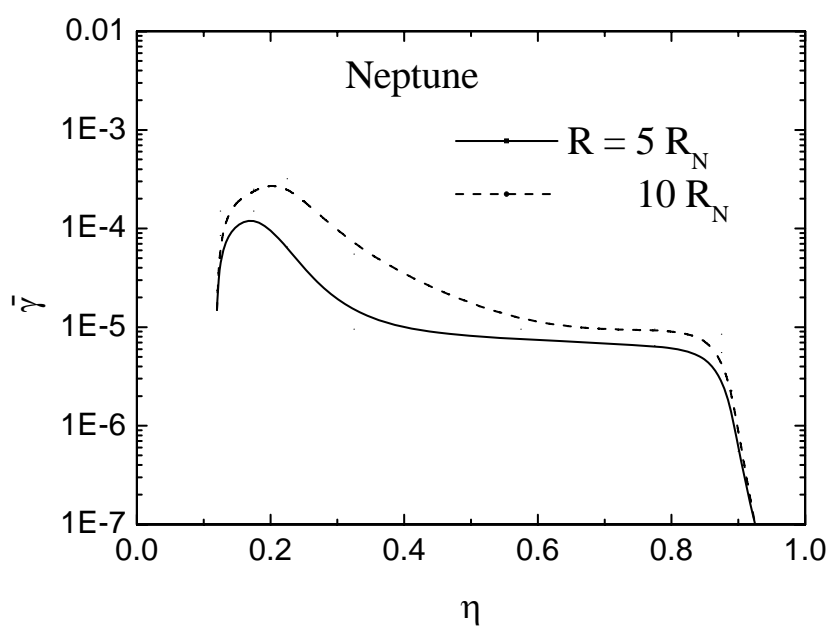

Fig. 5. Same as in Fig. 1 but for Neptune.

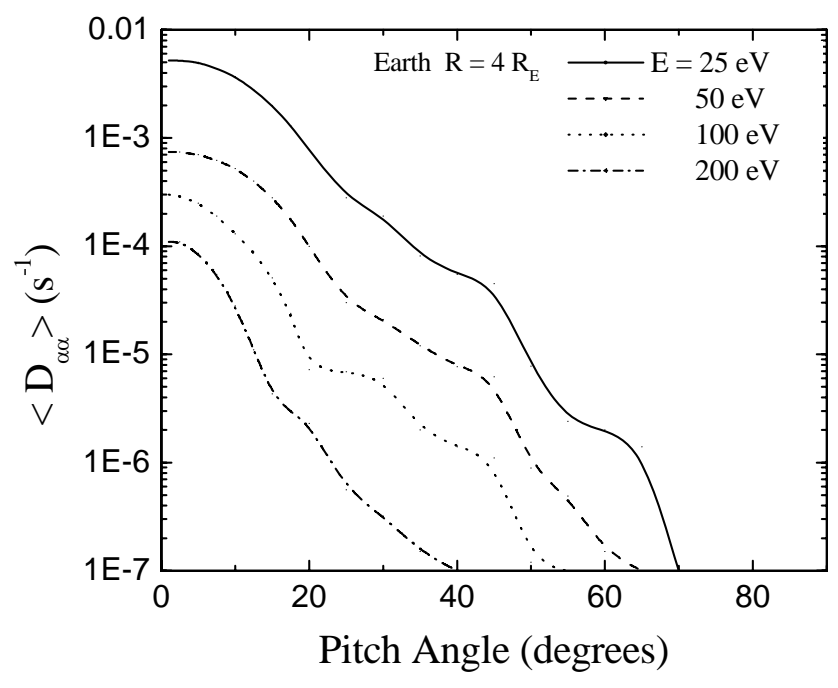

Fig. 6. Bounce-averaged pitch-angle diffusion coefficient versus equatorial pitch-angle for several electron energies for Earth at $R=4 R_{\mathrm{E}}$. Other parameters are $n=1, x_{\omega}=0.01$, and $E_{\text {Wave }}=$ $1 \mathrm{mV} \mathrm{m}^{-1}$.

particularly at higher distance. This is in agreement with the results reported by Horne and Thorne (2000). The diffusion rates for Uranus and Neptune are on the order of magnitude lower than that for Earth, Jupiter and Saturn. Table 2 presents the bounce-averaged diffusion and strong diffusion rates for several electron energies for two radial distances of each planet. The magnitude of the electric field of wave required for strong diffusion is given in the last column of Table 2. This table also presents the ratio of plasma frequency to cyclotron frequency $\left(\omega_{\mathrm{pe}} / \Omega_{\mathrm{ce}}\right)$ at two distances corresponding to each planet.

Comparing the diffusion rates with strong diffusion rates (given in Table 2) we note that on Earth and Jupiter, observed wave field may be sufficient for strong diffusion at

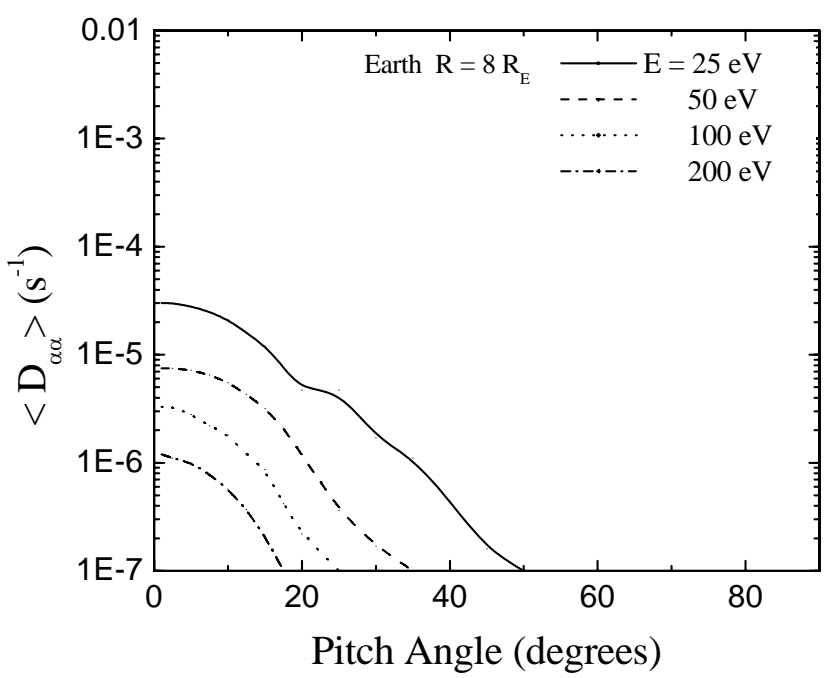

Fig. 7. Same as in Fig. 6 but for Earth at $R=8 R_{\mathrm{E}}$ and $E_{\text {Wave }}=$ $0.1 \mathrm{mV} \mathrm{m}^{-1}$.

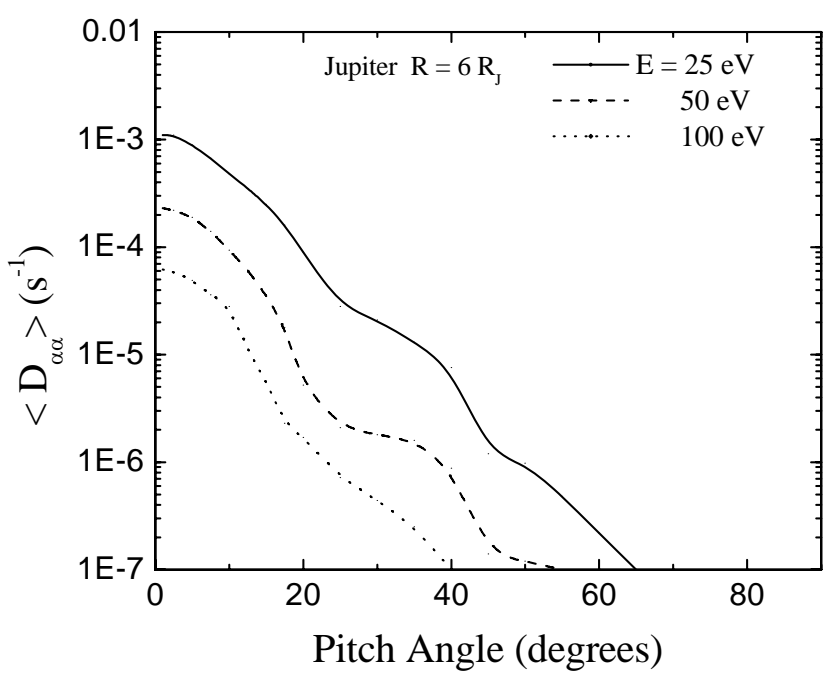

Fig. 8. Same as in Fig. 6 but for Jupiter at $R=6 R_{\mathrm{J}}$ and $E_{\text {Wave }}=$ $0.1 \mathrm{mV} \mathrm{m}^{-1}$.

two radial distances. However, for Saturn, Uranus and Neptune, observed wave field is not sufficient for strong pitch angle diffusion. Also at larger distance and for low energy electrons the observed wave field may be sufficient to put electrons on strong diffusion. Also at higher distance and low energy electrons the required wave field may be sufficient to put electrons on strong diffusion. Comparing the present results for Earth with the recent work reported by Horne and Thorne (2000) it may be noted that our results for the required amplitude of wave field for strong diffusion are in agreement with their results for electron energies of a few hundred eV. Studies of Meredith et al. (2000) suggest that $\mathrm{ECH}$ waves inside $L=6$ play a significant role in the production of diffuse aurora. Thus it may be concluded that 


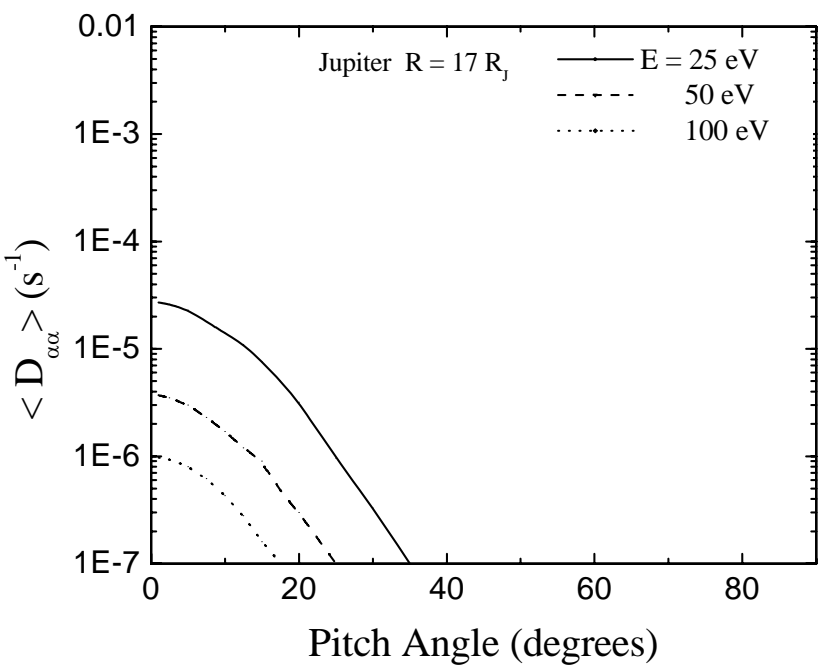

Fig. 9. Same as in Fig. 6 but for Jupiter at $R=17 R_{\mathrm{J}}$ and $E_{\text {Wave }}=$ $10 \mu \mathrm{V} \mathrm{m}^{-1}$.

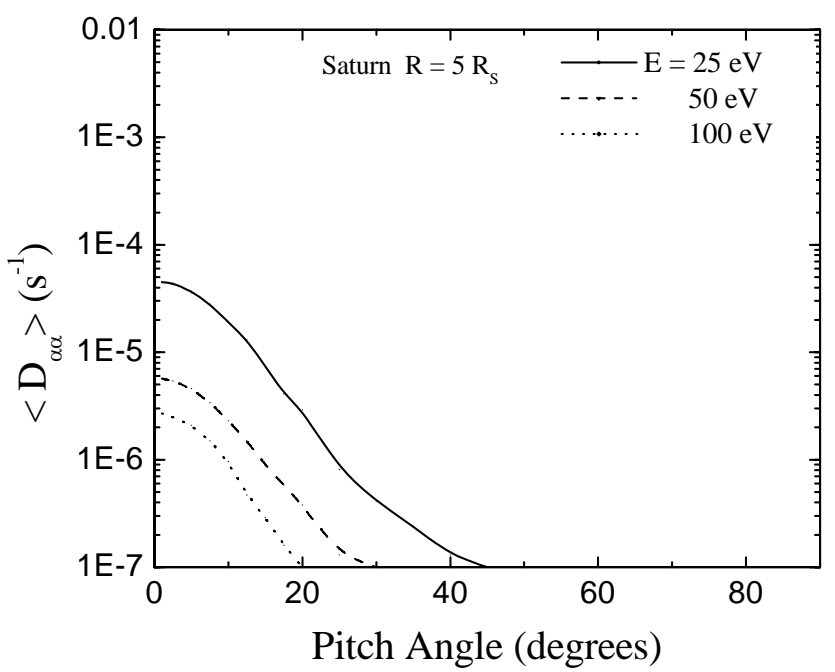

Fig. 10. Same as in Fig. 6 but for Saturn at $R=5 R_{\mathrm{S}}$ and $E_{\text {Wave }}=$ $30 \mu \mathrm{V} \mathrm{m}^{-1}$.

$\mathrm{ECH}$ waves are responsible for diffuse auroral precipitation of electrons with energies of a few hundred eV. However, in case of few $\mathrm{keV}$ electrons the observed $\mathrm{ECH}$ wave intensities are insufficient to cause electron precipitation into the terrestrial atmosphere. Resonant scattering of plasma sheet electrons $(\sim 100 \mathrm{eV}-20 \mathrm{keV})$ at $L=6$ due to resonant interaction with whistler-mode chorus has recently been presented by $\mathrm{Ni}$ et al. (2008). It has been concluded by these authors that scattering by chorus is more effective than resonant interaction with electrostatic electron cyclotron waves in producing diffuse aurora. In the present study we find that ECH waves can cause pitch-angle diffusion of electrons of energies of less than $100 \mathrm{eV}$. Thus a combination of both wave types $(\mathrm{ECH}$ and whistler-mode waves) may be necessary to cause diffuse

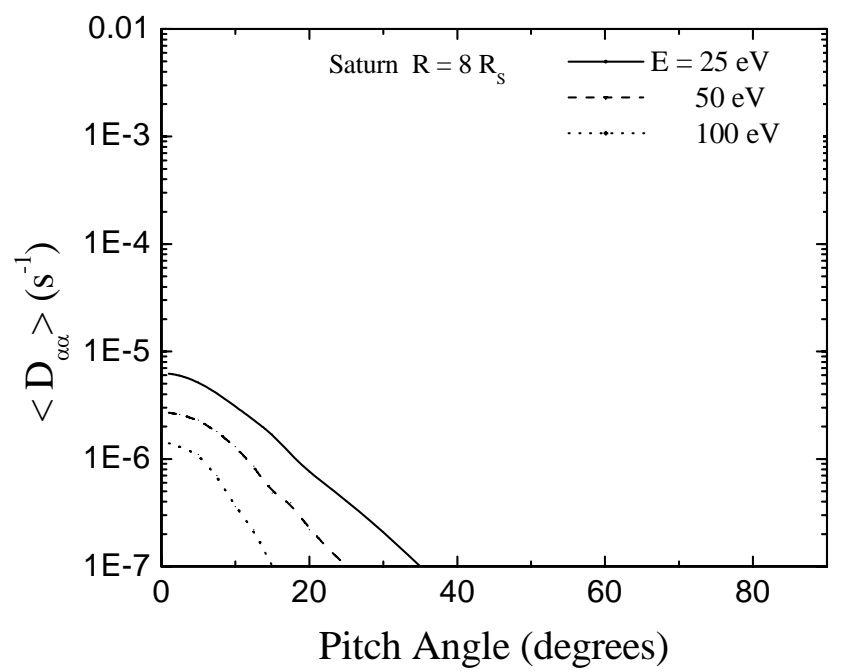

Fig. 11. Same as in Fig. 6 but for Saturn at $R=8 R_{\mathrm{S}}$ and $E_{\text {Wave }}=$ $10 \mu \mathrm{V} \mathrm{m}^{-1}$.

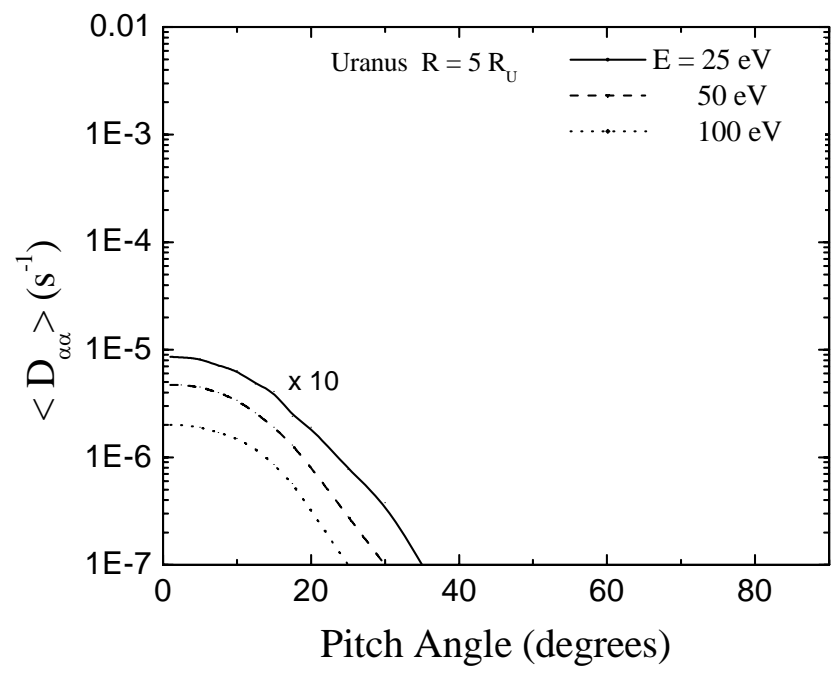

Fig. 12. Same as in Fig. 6 but for Uranus at $R=5 R_{\mathrm{U}}$ and $E_{\text {Wave }}=$ $30 \mu \mathrm{V} \mathrm{m}^{-1}$. Diffusion coefficient have been multiplied by 10 before plotting.

aurora (Meredith et al., 1999). For Jupiter, Thorne (1983) has indicated that observed wave intensity could derive a strong diffusion of low energy electrons. This is in agreement with the present results. However, Sittler and Strobel (1987) state that the electron data are not consistent with strong diffusion (Kurth and Gurnett, 1991). In the case of Saturn, it appears that the intensity of ECH waves is insufficient for strong pitch angle diffusion. Similarly for Uranus and Neptune, the observed low intensity of ECH waves is insufficient for strong pitch angle diffusion. It is however, possible that for Uranus at a lower radial distance, significant wave intensity could exist which has not been detected by Voyager. The same may be true for Neptune. It is possible that $\mathrm{ECH}$ waves of sufficient 


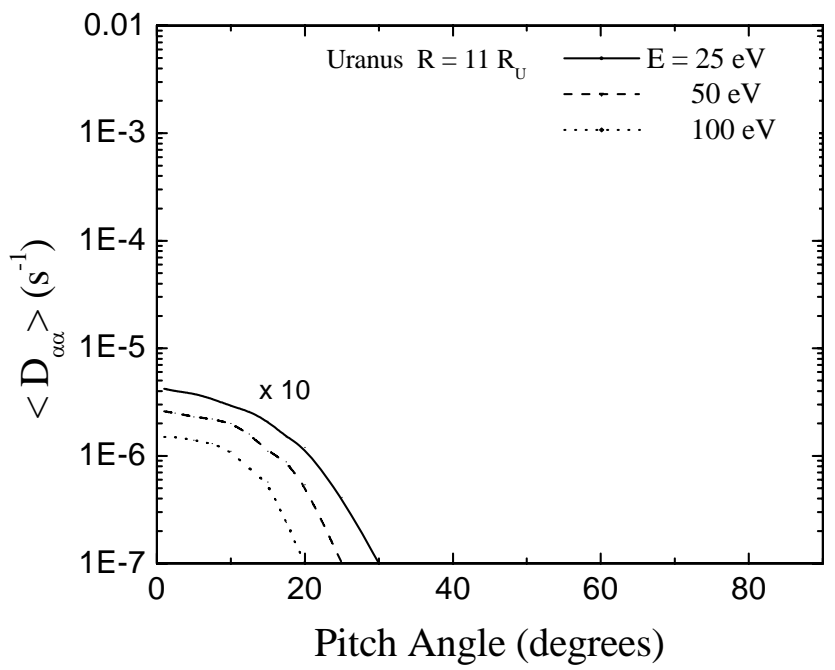

Fig. 13. Same as in Fig. 6 but for Uranus at $R=11 R_{\mathrm{U}}$ and $E_{\text {Wave }}=$ $10 \mu \mathrm{V} \mathrm{m}^{-1}$. Diffusion coefficient have been multiplied by 10 before plotting.

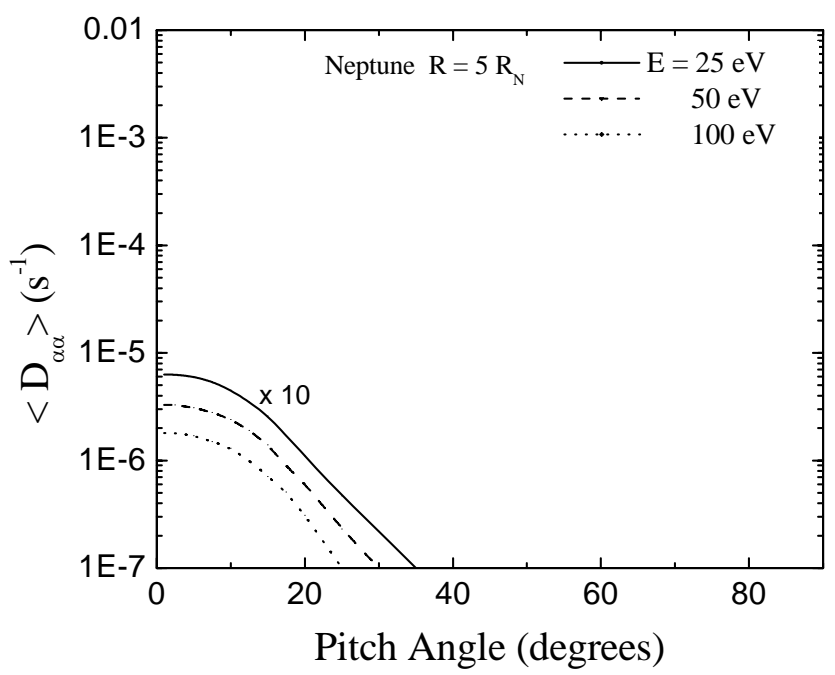

Fig. 14. Same as in Fig. 6 but for Neptune at $R=5 R_{\mathrm{N}}$ and $E_{\text {Wave }}=$ $30 \mu \mathrm{V} \mathrm{m}^{-1}$. Diffusion coefficient have been multiplied by 10 before plotting.

intensity for strong wave-particle interactions exist closer to Neptune and could comprise a significant loss-mechanism of energetic electrons in Neptunian magnetosphere. Kurth and Gurnett (1991) present a comparative study of ECH waves of the planetary magnetospheres and especially the assessment of their roles in precipitation of charged particles into planetary atmosphere. The waves seem to have obvious contributions in this way for Earth and Jupiter. Yet, the situations in the cases of Saturn, Uranus and Neptune are not better understood due to weaker wave amplitudes observed at these planets. The present results are in agreement with the study of Kurth and Gurnett (1991).

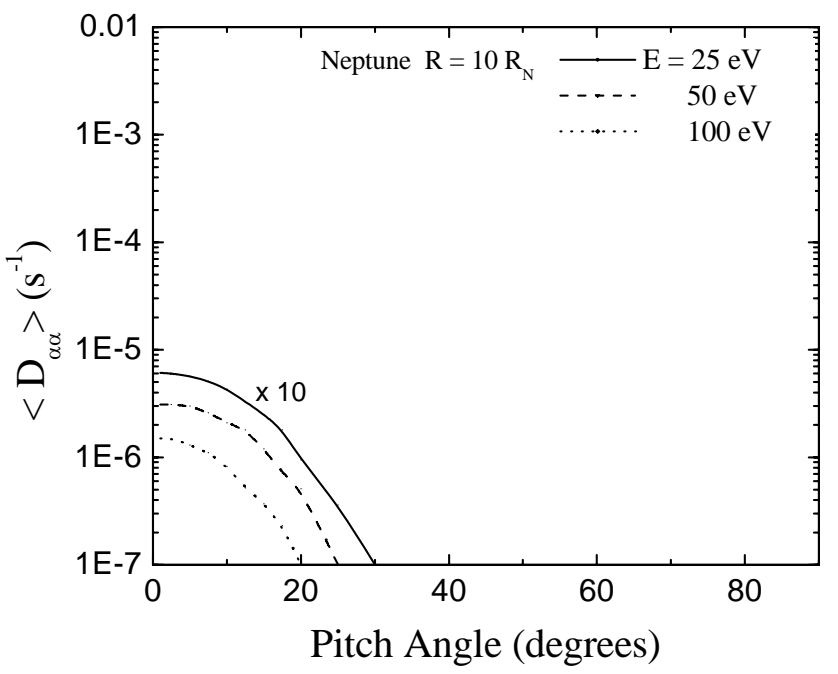

Fig. 15. Same as in Fig. 6 but for Neptune at $R=10 R_{\mathrm{N}}$ and $E_{\text {Wave }}=10 \mu \mathrm{V} \mathrm{m}^{-1}$. Diffusion coefficient have been multiplied by 10 before plotting.

\section{Conclusions}

In the present work for the first time we have calculated pitch angle diffusion coefficients for resonant interactions of electrons with ECH waves in the five planetary magnetospheres: Earth, Jupiter, Saturn, Uranus and Neptune. Further, we have compared the pitch-angle diffusion by ECH waves in these magnetospheres. The ECH wave intensity is expressed as a function of wave normal angle and wave frequency. Numerical results of the present calculations have been performed for the first harmonic band and the observed results are compared to the previous results. The main results obtained from this study are as follows.

1. In the case of Earth, only low energy electrons $(<100 \mathrm{eV})$ are sufficient to put electrons on strong diffusion at both lower and higher radial distances to produce diffuse aurora in the terrestrial atmosphere. For diffusion of higher energy electrons scattering by whistlermode the chorus may be more effective as suggested by Ni et al. (2008).

2. In the case of Jupiter, electrons of about $50 \mathrm{eV}$ may be sufficient to put electrons on strong diffusion at lower radial distance. However, at larger radial distance electrons of higher energy $(100 \mathrm{eV})$ may be put on strong diffusion.

3. The present study shows that for Saturn, Uranus and Neptune observed wave intensities are insufficient to put electrons on strong diffusion at both radial distance in magnetospheres. It may, however, be, noted that Voyager did not sample the ECH waves at smaller radial distances, where significant intensities could exist without having been detected. 
4. Thus, it may be concluded that for Earth and Jupiter, $\mathrm{ECH}$ waves can cause scattering of low energy electrons $(<100 \mathrm{eV})$ producing diffuse aurora but on Saturn, Uranus and Neptune the observed wave intensities are insufficient to cause diffuse aurora.

5. The present calculations are based on quasi-linear diffusion theory. Perhaps other mechanisms such as interaction by whistler-mode chorus are required to produce the diffuse aurora or the validity of quasi-linear diffusion theory needs to be investigated.

Acknowledgements. This work was supported by financial assistance provided by the Council of Scientific and Industrial Research, Government of India under a Senior Research Associateship (Scientist's Pool Scheme) award to one of the author (A.K.T). Calculations reported in the present work were carried out at the Computer Centre, Banaras Hindu University.

Topical Editor R. Nakamura thanks one anonymous referee for her/his help in evaluating this paper.

\section{References}

Bagenal, F.: Empirical model of the Io plasma torus: Voyager measurements, J. Geophys. Res., 99, 11043-11062, 1994.

Bagenal, F., Crary, F. J., Stewart, A. I. F., Schneider, N. M., Gurnett, D. A., Kurth, W. S., Frank, L. A., and Peterson, W. R.: Galileo measurements of plasma density in the Io torus, Geophys. Res. Lett., 24, 2119-2122, 1997.

Belcher, J. W.: The low-energy plasma in Jovian magnetosphere, in: Physics of the Jovian Magnetosphere, Cambridge University Press, New York, 105 pp., 1983.

Belcher, J. W., Bridge, H. S., Bagenal, B., Coppi, B., Divers, O., Eviator, A., Gardon, G. S. Ir., Lazarus, A. J., McNutt Jr., R. L., Ogilvie, K. W., Richardson, J. D., Siscoe, G. L., Sittler Jr., E. C., Steinberg, J. T., Sullivan, J. D., Szabo, A., Villanueva, L., Vasyliunas, V. M., and Zhang, M.: Plasma observations near Neptune: initial results from Voyager 2, Science, 246, 1478-1483, 1989.

Décréau, P. M. E., Fergeau, P., Krasnoselskikh, V., Le Guirriec, E., Lévêque, M., Martin, Ph., Randriamboarison, O., Rauch, J. L., Sené, F. X., Séran, H. C., Trotignon, J. G., Canu, P., Cornilleau, N., de Féraudy, H., Alleyne, H., Yearby, K., Mögensen, P. B., Gustafsson, G., André, M., Gurnett, D. C., Darrouzet, F., Lemaire, J., Harvey, C. C., Travnicek, P., and Whisper experimenters: Early results from the Whisper instrument on Cluster: an overview, Ann. Geophys., 19, 1241-1258, doi:10.5194/angeo-19-1241-2001, 2001.

Fredricks, R. W. and Scarf, F. L.: Recent studies of magnetospheric electric field emissions above the electron gyrofrequency, J. Geophys. Res., 78, 310-314, 1973.

Gurnett, D. A., Anderson, R. R., Scarf, F. L., Fredricks, R. W., and Smith, E. J.: initial results from the ISEE-1 and -2 plasma wave investigation, Space Sci. Rev., 23, 103-122, 1979a.

Gurnett, D. A., Kurth, W. S., and Scarf, F. L.: Plasma wave observations near Jupiter: initial results from voyager 2, Science, 206, 987-991, 1979b.

Gurnett, D. A., Kurth, W. S., and Scarf, F. L.: Plasma wave near Saturn: initial result from Voyager 1, Science, 212, 235-239, 1981.
Gurnett, D. A. and Inan, U. S.: Plasma waves observations near with Dynamics explorer 1 spacecraft, Rev. Geophys., 26, 285316, 1988.

Gurnett, D. A., Kurth, W. S., and Poynter, R. L.: first plasma waves observations at Neptune, Science, 246, 1494-1498, 1989.

Gurnett, D. A., Kurth, W. S., and Roux, A.: Galileo plasma wave observations in the Io plasma torus and near Io, Science, 274, 391-392, 1996.

Gurnett, D. A., Kurth, W. S., and Hospodersky, G. B.: Radio and plasma wave observations at Saturn from Cassinis approach and first orbit, Science, 307, 1255-1259, 2005.

Horne, R. B. and Thorne, R. M.: Electron pitch-angle diffusion bt electrostatic electron cyclotron harmonic waves: the origin of pancake distributions, J. Geophys. Res., 105, 5391-5402, 2000.

Horne, R. B., Thorne, R. M., Meredith, N. P., and Anderson, R. R.: Diffuse auroral electron scattering by electron cyclotron harmonic and whistler mode waves during an isolated substorm, J. Geophys. Res., 108(A7), 1290, doi:10.1029/2002JA009739, 2003.

Kennel, C. F. and Petschek, H. E.: Limit on stably trapped particle fluxes, J. Geophys. Res., 71, 1-29, 1966.

Kennel, C. F., Scarf, F. L., Fredricks, R. W., McGehee, J. H., and Coroniti, F. V.: VLF electric field observations in the magnetosphere, J. Geophys., Res, 75, 6136-6152, 1970.

Kurth, W. S., Barbosa, D. D., Gurnett, D. A., and Scarf, F. L.: Electrostatic waves in the Jovian magnetosphere, Geophys Res. Lett., 7, 57-60, 1980.

Kurth, W. S., Scarf, F. L., Gurnett, D. A., and Barbosa, D. D.: A survey of electrostatic waves in Saturns magnetosphere, J. Geophys. Res., 88, 8959-8970, 1983.

Kurth, W. S., Barbosa, D. D., Gurnett, D. A., and Scarf, F. L.: electrostatic waves in magnetosphere of Uranus, J. Geophys. Res., 92, 15225-15233, 1987.

Kurth, W. S. and.Gurnett, D. A.: Plasma wave observations in planetary magnetosphere, J. Geophys. Res., 96, 18977-18991, 1991.

Laakso, H., Pfaff, R., and Janhunen, P.: Polar observations of electron density distribution in the Earths magnetosphere. 2. Density profiles, Ann. Geophys., 20, 1725-1735, doi:10.5194/angeo-201725-2002, 2002.

Lyons, L. R.: Electron diffusion driven by magnetospheric electrostatic waves, J. Geophys. Res., 79, 575-580, 1974.

Meredith, N. P., Johnstone, A. D., Szita, S., Horne, R. B., Anderson, R. R.: Pancake electron distributions in the outer radiation belts, J. Geophys. Res., 104, 12431-12444, 1999.

Meredith, N. P., Horne, R. B., Johnstone, A. D., Anderson, R. R.: the temporal evolution of electron distributions and associated wave activity following substorm injection in the inner magnetosphere, J. Geophys. Res., 105, 12907-12917, 2000.

Ni, B., Thorne, R. M., Shprits, Y. Y., and Bortnik, J.: Resonating scattering of plasma sheet electrons by whistler-mode chorus: contribution to diffuse auroral precipitation, Geophys. Res. Lett., 35, L11106, doi:10.1029/2008GL034032, 2008.

Olsen, R. L., Shawhan, S. D., Gallagher, D. L., Green, J. L., Chappell, C. R., and Anderson, R. R.: Plasma observations at the earths magnetic equator, J. Geophys Res., 92, 2385-2407, 1987.

Richardson, J. and Sittler Jr., E. C.: A plasma density model for Saturn based on Voyager observstions, J. Geophys. Res., 95, 1201912031, 1990.

Scarf, F. L., Gurnett, D. A., and Kurth, W. S.: Jupiter plasma wave 
observations: An initial Voyager 1 overview, Science, 204, 991995, 1979.

Scarf, F. L., Gurnett, D. A., Kurth, W. S., and Poynter, R. L.: Voyager 2 plasma waves observations at Saturn, Science, 215, 587594, 1982.

Shaw, R. R. and Gurnett, D. A.: Electrostatic noise bands associated with the electron gyrofrequency and plasma frequency in the outer magnetosphere, J. Geophys. Res., 80, 4259-4271, 1975.

Sittler Jr., E. C. and Strobel, D. F.: Io plasma torus electron: Voyager 1, J. Geophys. Res., 92, 5741-5762, 1987.

Sittler Jr., E. C., Ogilive, K. W., and Scudder, J. D.: Survey of lowenergy plasma electrons in Saturn's Magnetosphere: Voyager 2 observations, J. Geophys. Res., 88, 8847-8870, 1983.

Sittler Jr., E. C., Ogilive, K. W., and Selesnick, R.: Survey of electrons in the Uranian magnetosphere, Voyager 2 observations, J. Geophys. Res., 92, 15263-15281, 1987.

Stone, R. G., Pedersen, B. M., Harvey, C. C., Canu, P., CornilleauWehrlin, N., Desch, M. D., deVilledary, C., Fainberg, J., Farrell, W. M., Geoetz, K., Hess, R. A., Hoang, S., Kaiser, M. L., Kel$\operatorname{logg}$, P. J., Lecacheux, A., Lin, N., MacDowell, R. J., Manning, R., Meetre, C. A., Meyer-Vernet, N., Moncuqet, M., Ocherovich, V., Reiner, M. J., Tekle, A., Thiessen, J., and Zarka, P.: Ulysses radio and plasma wave observations in Jupiter environment, Science, 257, 1524-1531, 1992.
Summers, D. and Thorne, R. M.: plasma microinstabilities driven by loss-cone distributions, J. Plasma Phys., 53, 293-315, 1995.

Summers, D., Ni, B., and Meredith, N. P.: Timescales for radiation belt electron acceleration and loss due to resonant wave-particle interactions: 2. Evalution for VLF chorus, ELF hiss, and electromagnetic ion-cyclotron waves, J. Geophys. Res., 112, A04207, doi:10.1029/2006JA011993, 2007.

Summers, D., Tang, R., and Thorne, R. M.: Limit on stably trapped particle fluxes in planetary magnetosphere, J. Geophys. Res., 114, A10210, doi:10.1029/2009JA014428, 2009.

Thorne, R. M.: Microscopic plasma processes in the Jovian magnetosphere, in: Physics of the Jovian magnetosphere, edited by: Dessler, A. J., Cambridge, Univ. press, Cambridge, p. 454, 1983.

Tripathi, A. K. and Singhal, R. P.: diffusion coefficients from resonant interactions with electrostatic cyclotron harmonic waves, Phys. Plasmas, 16, 112107, doi:10.1063/1.3264735, 2009.

Zarka, P.: Radio and plasma waves at the outer planets, Adv. Space Res., 33, 2045-2060, 2004.

Zhang, M., Richardson, J. D., and Sittler Jr., E. C.: Voyager 2 electron observations in the magnetosphere of Neptune, J. Geophys. Res., 96, 19085-19100, 1991. 